\title{
Enhancing OPAC Records for Discovery
}

This article proposes adding keywords and descriptors to the catalog records of electronic databases and media items to enhance their discovery. The authors contend that subject liaisons can add value to OPAC records and enhance discovery of electronic databases and media items by providing searchable keywords and resource descriptions. The authors provide an examination of OPAC records at their own library, which illustrates the disparity of useful keywords and descriptions within the notes field for media item records versus electronic database records. The authors outline methods for identifying useful keywords for indexing OPAC records of electronic databases. Also included is an analysis of the advantages of using Encore's Community Tag and Community Review features to allow subject liaisons to work directly in the catalog instead of collaborating with cataloging staff.

A $\mathrm{t}$ the University of Nevada Las Vegas (UNLV) Libraries' Discovery Mini-Conference, there was a wide range of initiatives and ideas presented. Some were large-scale initiatives that focused on designing search platforms and systems as well as information architecture schemas that would enhance library resource discovery. But there was not much focus on enhancing the representation of library resources within the construct of bibliographic records in the OPAC. Since searching platforms can only be as useful as the information available for searching, and since OPAC records are the method for representing the majority of library resources, we thought it important that the prominence of OPAC records and how they represent library resources be considered in the Mini-Conference. To that end, our presentation focused on enhancing the OPAC records for nonbook items to support their discoverability as opposed to focusing on search systems and information architecture schemas. Our proposition was that subject liaisons' expertise could be used to enhance OPAC records by including their own keyword search terms and descriptive summaries in OPAC records for electronic databases as well as records of media items.

This proposition acts as a moderate approach to initiatives that call for OPAC records to be opened for usergenerated content in that this approach provides subject liaison mediation and expertise to modify records. As such, this approach may serve as an effective stopgap in

Patrick Griffis (patrick.griffis@unlv.edu) is Business Librarian and Cyrus Ford (cyrus.ford@unlv.edu) is Special Formats Catalog Librarian, University of Nevada Las Vegas Libraries.

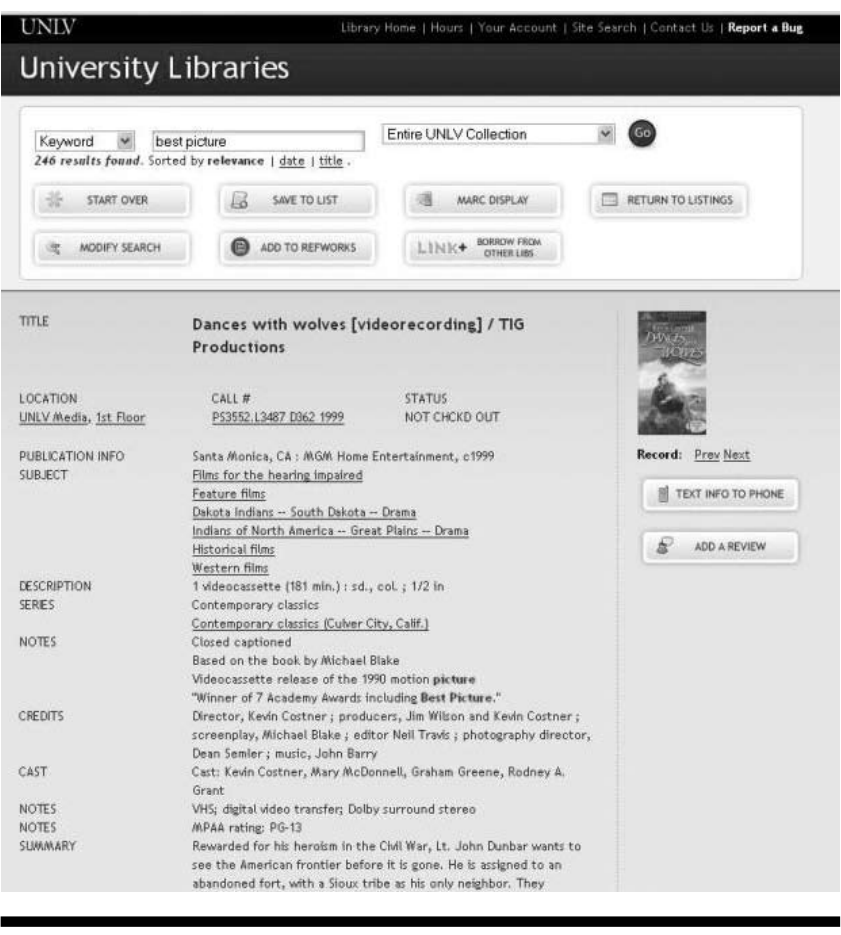

Figure 1. The notes field in an OPAC record of a film item

cases where there is resistance toward permitting social tagging and user descriptions within OPAC records. Such an initiative also is scalable, allowing liaisons to provide as few or as many terms as they want. Such an initiative would require collaboration between cataloging staff and subject liaisons.

\section{Disparity between media and database records}

At UNLV Libraries, terms included in the notes fields of bibliographic records are indexed for keyword searching. In the case of media items, there is extensive use of notes to include descriptive terms that enhance discoverability for users. For example, notes for films indicate any awards the film has won as well as festivals in which it has been featured (see figure 1). As a result, users can discover films through keyword searches of film awards or film festivals. A film student who is searching "Cannes Film Festival" via a keyword search will generate results that include films owned by UNLV Libraries that have been featured at that festival. These keyword-searchable notes add value and discoverability for this type of material, and subject liaisons can be a source for such information.

While it appears that notes in media records are heavily populated with a variety of user-centric information, there is relatively little use of descriptive notes for 


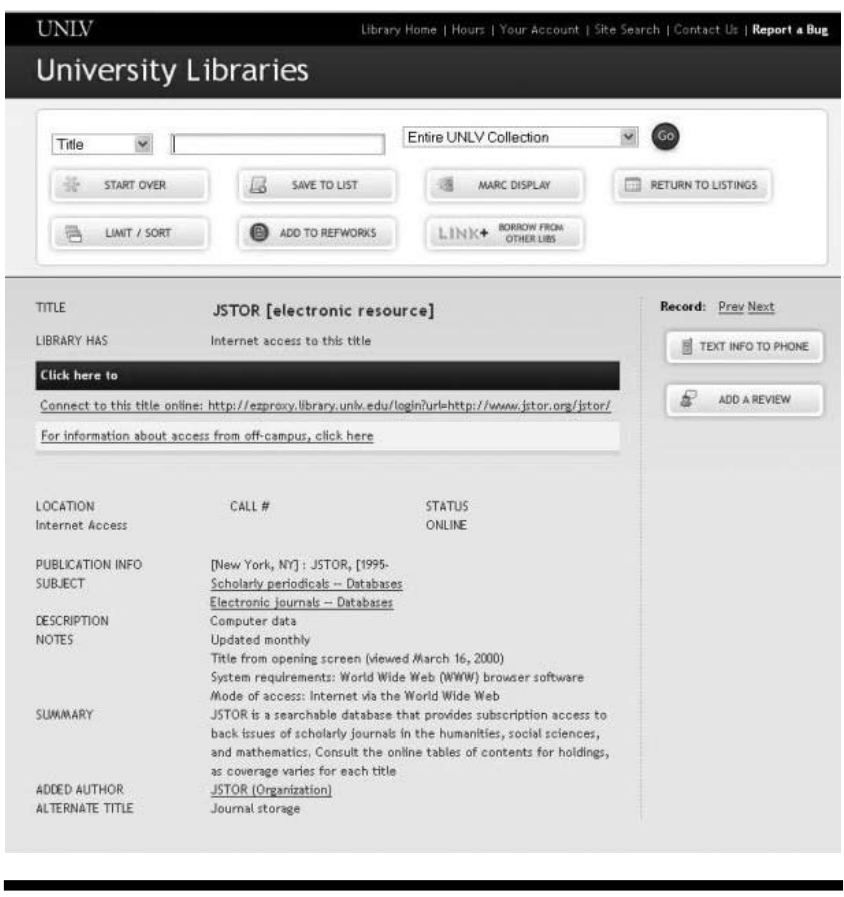

Figure 2. The notes field in an OPAC record of an electronic database

electronic databases (see figure 2). For databases, notes traditionally include information about access restrictions and mode of access while overlooking information representing the content of the resource. These fields could be utilized for specific terms relating to database content not adequately covered by the Library of Congress Subject Headings (LCSH). Subject liaisons have specialized knowledge of which databases work best for unique content areas, class assignments, and information needs. This user-centric knowledge can be used to enhance database discovery if liaisons were to provide catalogers with information and descriptors to add to the record. As an example, at UNLV Libraries there is one particular database that provides a Strengths, Weaknesses, Opportunities, and Threats (SWOT) analysis for companies, but that natural language term isn't found anywhere in the general database summary listing or subject headings. If it were added to a note field as part of a description or as a labeled descriptor, then students could easily find this database to complete their assignments.

This proposal is scalable, allowing liaisons to provide as few or as many key terms as they want, depending on their preference or on the vagaries of a particular database. Subject liaisons could opt to add a few major terms from their own knowledge and expertise that they feel will add value for patrons searching the OPAC. Subject liaisons also could mine the index and thesaurus terms of individual databases to identify prominent content areas for individual databases to find useful keywords.

\section{Mining electronic database index descriptors}

Electronic databases typically have subject matter taxonomies developed by experts who assign descriptors to journal articles. Subject liaisons could mine these taxonomies to identify predominant descriptors for individual databases to add to the database catalog records. Predominance of a subject descriptor could be determined by examining the relative number of articles that are assigned to that descriptor. Such a strategy of indexing key predominant subject descriptors identified from database subject matter taxonomies could serve to uncover unique content areas not served with LCSH.

A different application of this strategy could be employed for identifying predominant and emerging research areas for particular groups. Subject liaisons could conduct a citation analysis of articles authored by members of a particular research group to record and codify the subject descriptors of each article. Once codified, an analysis could determine the most predominant subject descriptors for articles authored by that particular group. This could serve as a baseline for identifying emerging research areas and their terms. Both types of analysis have potential to provide useful keyword terms for database records.

\section{Using Encore's community features}

In 2008, UNLV Libraries purchased and implemented the Innovative Interfaces' Encore discovery platform, which provides a Google-like interface for searching the public catalog and the ability to narrow results using facets such as location, year, language, and format. Encore also includes many display features that showcase the information provided in the bibliographic records. Two of Encore's Web 2.0 features provide users with the ability to contribute data to records via community tags and community reviews. UNLV requires users to enter a valid library barcode number and PIN. Subject liaisons could use the community reviews feature to add descriptive summaries of items to Encore records independently, without the need for cataloging staff to edit a MARC record. However, the content of community reviews are not indexed for searching and thus only add value at the point when a user is determining whether the resources they have retrieved are valuable for them.

On the other hand, if a community tag is added to an item, that tag is included in the community tags section 


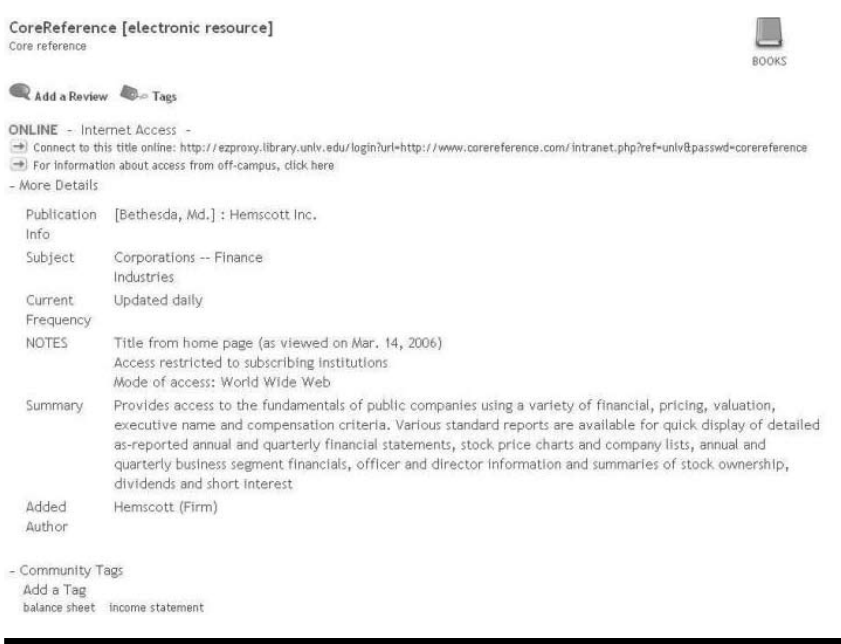

Figure 3. Encore community tag

of the Encore result display and becomes an indexed keyword for searches in Encore (see figure 3). If that tag term is searched in Encore's keyword search, the bibliographic record attached to that tag term will be included in the results list under the community tags facet. Since these community tags are searchable, subject liaisons can add keywords to Encore records without collaboration with cataloging staff. However, this provides limited success because the keyword is included and indexed only in Encore records-not in the OPAC records. Also, the community tags facet must be selected from the results display for the Encore record tags to be searchable.

\section{The case for collaboration}

As described above, keywords and descriptions added by subject liaisons into Encore records have inherent discovery limitations when compared to a cataloger adding the same information directly to the MARC bibliographic record. The advantages of collaboration between subject liaisons and catalogers is clear, and subject librarians at UNLV Libraries have experienced similar collaboration in efforts in the past.

In 2006, subject librarians at UNLV Libraries were offered the opportunity to create their own descriptions of electronic resources through an initiative to update the summary descriptions for the electronic databases portion of the Libraries' website. At that time, all existing electronic database summaries were those used by the database publishers. The project provided subject liaisons the option to create custom summary descriptions to represent electronic databases in their own terms. Each subject liaison had a document file for their descriptions, and the website editors used them to update the electronic databases list on the Libraries' website.

This particular initiative serves as one example of the willingness of subject liaisons to share their subject expertise to enhance the representation of library resources through collaboration with Technical Services staff. As such, collaboration between subject liaisons and catalogers to allow liaisons to add terms to OPAC records of electronic databases and media items could prove to be both effective and feasible as an initiative toward enhancing the discovery of library resources.

\section{Index to Advertisers}

